\title{
Fluoxetine improves motor function after acute ischemic stroke
}

T he antidepressant fluoxetine, when given soon after an ischemic stroke, improves motor recovery and could help patients with stroke maintain an independent lifestyle, according to a new double-blind randomized controlled study. "Affected motor function was significantly improved in patients on fluoxetine when compared with placebo," notes first author François Chollet, Hôpital Purpan, France. "The gain [in motor function] is about $50 \%$ increased recovery."

Muscle weakness or total paralysis on one side of the body is common after stroke. Results from a few small clinical trials have already suggested that poststroke administration of fluoxetine or other selective serotonin reuptake inhibitors (SSRIs) could have positive effects on movement. Moreover, functional MRI has shown that a single dose of fluoxetine stimulates-and can even overactivate-the motor cortices compared with placebo in both healthy individuals and patients who have experienced a stroke. Chollet and colleagues, therefore, wanted to test the clinical efficacy of fluoxetine in stroke patients with motor deficits.

In the fluoxetine for motor recovery after acute ischemic stroke (FLAME) trial, the researchers enrolled 118 patients with hemiparesis or hemiplegia after ischemic stroke from nine stroke centers across France. Individuals were randomly assigned in a 1:1 ratio to receive one daily dose of $20 \mathrm{mg}$ fluoxetine or placebo for 3 months starting within 5-10 days after stroke onset. All patients received physiotherapy regardless of treatment group assignment.

Only trial participants aged $18-85$ years with a Fugl-Meyer motor scale (FMMS) score $\leq 55$ were included; patients with severe disability before and after stroke and those who were clinically depressed were excluded from the study. The primary end point was the change in FMMS score at the end of the study period.

After adjusting for center, age, history of stroke, and FMMS score at inclusion, the improvement in FMMS score over 90 days was, on average, significantly higher in the fluoxetine group than the placebo group (34 points versus 24.3 points, $P=0.003$ ). The gain in motor function was substantial in both the upper and lower limbs.

Independence in activities of daily life (measured by the modified Rankin scale [mRS]) improved in both treatment groups after 3 months, but the proportion of independent patients who returned to a normal daily life (mRS score $0-2$ ) was markedly greater in the fluoxetine group (26\%) than in the placebo group (9\%). In addition, depression, which is common in stroke patients, was considerably reduced with fluoxetine therapy.

In terms of safety, fluoxetine seemed to be well tolerated in stroke patients, although patients receiving fluoxetine tended to have more incidents of

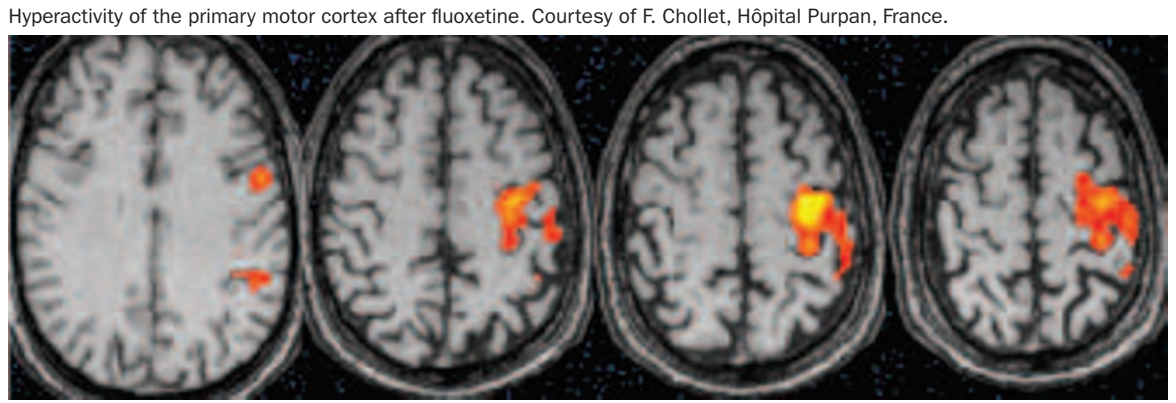

transient digestive disorders (nausea, diarrhea and abdominal pain) than those who received placebo.

The FLAME trial is, to date, the largest study to investigate the effects of SSRIs, and specifically fluoxetine, on stroke recovery. "I believe the findings have important implications for patient care, specifically suggesting that the use of this drug-and perhaps other antidepressants as well-administered early after stroke could improve motor recovery," says Leonardo Cohen from the National Institute of Neurological Disorders and Stroke, USA, who was not involved in the FLAME study.

More research is needed to determine the mechanisms that underlie the beneficial effects of fluoxetine on motor function in stroke patients, a sentiment shared by Cohen. "It remains to be determined if the results reported here [in the FLAME trial] reflect effects of the drug on mood and/or depression, rather than direct effects on motor neuroplasticity," he adds.

The study authors acknowledge that they have "no idea of the long-term development of the patients' motor function and whether the treatment effect persisted in the months after treatment was stopped." Chollet believes that the use of fluoxetine in the general stroke population warrants further investigation-the FLAME trial had strict inclusion criteria and was not representative of all patients with stroke-as does the optimal time for fluoxetine therapy and its effects on other neurological functions, such as language and vision.

Katrina Ray

Original article Chollet, F. et al. Fluoxetine for motor recovery after acute ischaemic stroke (FLAME): a randomised placebo-controlled trial. Lancet Neurol. 10 , 123-130 (2011) 